\title{
- Robots Have Needs Too: How and Why People Adapt Their Proxemic Behavior to Improve Robot Social Signal Understanding
}

\author{
Ross Mead and Maja J. Matarić \\ Interaction Lab, University of Southern California
}

\begin{abstract}
Human preferences of distance (proxemics) to a robot significantly impact the performance of the robot's automated speech and gesture recognition during face-to-face, social human-robot interactions. This work investigated how people respond to a sociable robot based on its performance at different locations. We performed an experiment in which the robot's ability to understand social signals was artificially attenuated by distance. Participants $(N=180)$ instructed the robot using speech and pointing gestures, provided proxemic preferences before and after the interaction, and responded to a questionnaire. Our analysis of questionnaire responses revealed that robot performance factors - rather than human-robot proxemics-are significant predictors of user evaluations of robot competence, anthropomorphism, engagement, likability, and technology adoption. Our behavioral analysis suggests that human proxemic preferences change over time as users interact with and come to understand the needs of the robot, and those changes improve robot performance.
\end{abstract}

Keywords: human-robot interaction, sociable robots, proxemics, social signals

\section{Introduction and Background}

A sociable robot utilizes natural communication mechanisms, such as speech and gesture, to autonomously interact with humans to accomplish some individual or joint task (Breazeal, 2004). Because the majority of human-robot interaction (HRI) contexts investigated to date involve oneon-one, face-to-face interaction between the robot and the human user, how the robot understands and responds to user input is crucial to autonomous sociable robots (Breazeal 2003).

Proxemics, the use of space (distance and orientation) in face-to-face encounters (Hall, 1959), is one of the most fundamental and natural human social behaviors. Accordingly, mobile sociable robots must use space appropriately when interacting with people. The position of a robot relative to a social partner has a significant impact on robot system performance - in this work, performance is measured by the robot's automated speech and gesture recognition rates. Just like electrical signals, human social signals (e.g., speech and gesture) are attenuated (lose signal strength) based on distance, directly impacting on-board automated signal detection and identification. Consequently, proxemic control systems that move the robot can also cause signal attenuation, therefore significantly influencing the success or failure of sociable robots (Mead \& Matarić, 2016).

\footnotetext{
Authors retain copyright and grant the Journal of Human-Robot Interaction right of first publication with the work simultaneously licensed under a Creative Commons Attribution License that allows others to share the work with an acknowledgement of the work's authorship and initial publication in this journal.
}

Journal of Human-Robot Interaction, Vol. 5, No. 2, 2016, Pages 48-68. DOI 10.5898/JHRI.5.2.Mead 
Anthropologist Edward T. Hall (1959) coined the term "proxemics," and, in Hall (1963), proposed that proxemics lends itself well to being analyzed through sensory experience via human perceptual systems. Proxemics has been studied in a variety of ways in HRI; this work, and thus this review, focuses on autonomous HRI system $\$ 1$

The majority of proxemics research in autonomous HRI addresses maximizing user preferences (or comfort) during face-to-face interaction. The results of many human-robot proxemics studies have been consolidated and normalized in Walters et al. (2009), reporting preferred mean distances of $0.49-0.71$ meters using a variety of robots and conditions. Proxemic preferences between humans and the PR2 robot were investigated in Takayama and Pantofaru (2009), reporting mean distances of 0.25-0.52 meters; in Mead and Matarić (2016), we investigated the same preferences using the PR2 in a conversational context, reporting a mean distance of 0.94 meters. Farther proxemic preferences have been measured in Mumm and Mutlu (2011) and Torta, Cuijpers, and Juola (2013), reporting mean distances of 1.0-1.1 meters and 1.7-1.8 meters, respectively.

However, our previous work indicates that automated robot speech and gesture recognition systems do not perform well (i.e., often fail) when using human preference-based proxemic configurations (Mead \& Matarić, 2016). Speech recognition performs adequately at distances of less than 2.5 meters, and face and hand gesture recognition performs adequately at distances of 1.5-2.5 meters; thus, given current technologies, distances for mutual recognition of these social signals is between 1.5 and 2.5 meters, a range at and beyond the far end of preference-based human-robot proxemics.

In Mead and Matarić (2016), we modeled sociable robot performance attenuated by distance and used the model to implement an autonomous robot proxemic controller that maximizes its performance during face-to-face HRI. While our approach to proxemic control improved the performance of the robot, it also resulted in proxemic configurations that are atypical for human-robot interactions (e.g., positioning the robot farther from or nearer to the user than preferred). This existence of conflicting evidence highlights the question of whether people would adopt a technology that places performance over preference, as it might require them to change their own behaviors to make the technology function adequately. This question is the focus of the work reported here.

This work investigates 1) how user proxemic preferences change (based on behavioral measures) in the presence of a sociable robot that is recognizing and responding to instructions provided by a human user, and 2) how users evaluate (based on subjective measures) the robot as its performance varies in different proxemic configurations. We artificially attenuated the robot's performance (i.e., ability to understand speech and gesture) to expose participants to success and failure scenarios during interactions with the robot. The next section describes the experimental setup. The specific experiment procedures, conditions, and hypotheses are outlined in Sections 3,4 and 5 , respectively. An analysis of behavioral and subjective results is presented in Section 6 and discussed in Section 7 Limitations of the approach are detailed in Section 8 and conclusions are provided in Section 9

\section{Experimental Setup}

\subsection{Materials}

The experimental robotic system used in this work was the Bandit upper-body humanoid robot (Fig. 11). Bandit has 19 degrees of freedom (DOFs): 7 in each arm (2 in each of the shoulders, elbows, and wrist, and 1 in each grabber), 2 in the head (pan and tilt), 2 in the lips, and 1 in the eyebrows. These DOFs allow Bandit to be expressive using individual and combined motions of the head, face, and arms. Mounted atop a Pioneer 3-AT mobile base 4 , the robot is 1.3 meters tall.

\footnotetext{
${ }^{1}$ How humans react and adapt to various technologies is beyond the scope of this work. For a review, see Kahn (2011).

2 https://www.willowgarage.com/pages/pr2/overview

3 http://robotics.usc.edu/interaction/? l=Laboratory: Robots\#Bandit I I

4 http://ww. mobilerobots.com/ResearchRobots/P3AT.aspx
} 


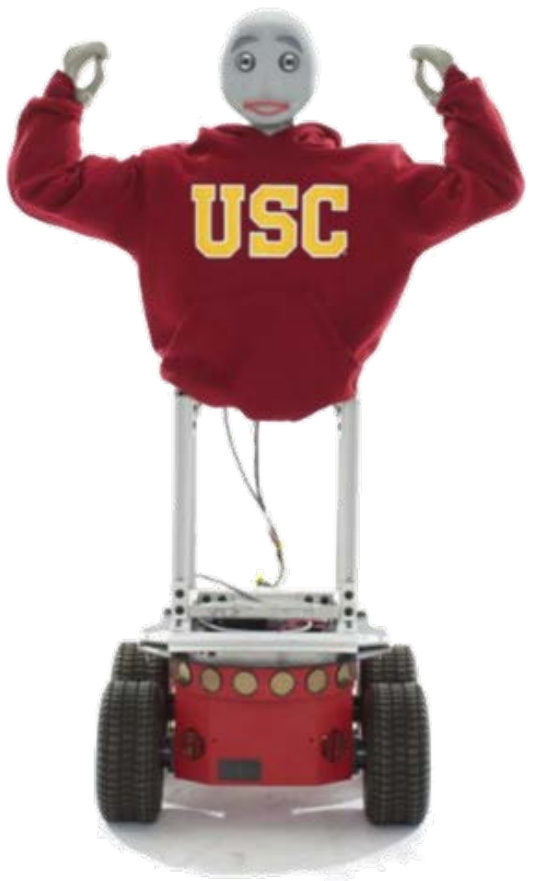

Figure 1. Bandit upper-body humanoid robot platform mounted atop a Pioneer 3-AT mobile base.

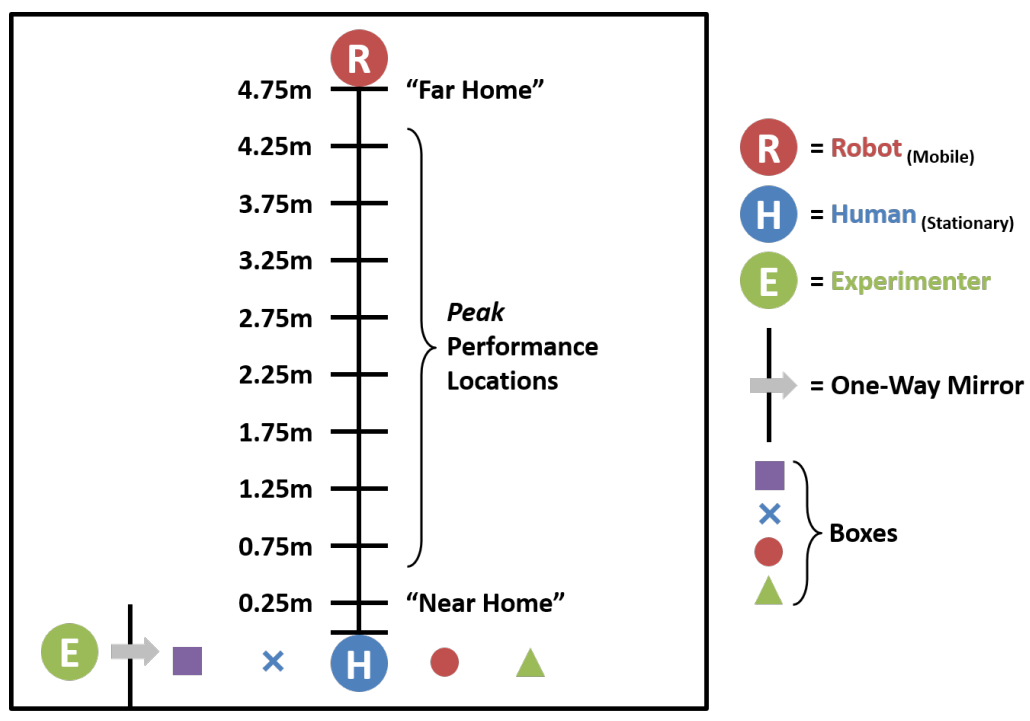

Figure 2. Experimental setup for evaluating human responses to robot behaviors. 
The experimenter (seated behind a one-way mirror; Fig. 2) used a Bluetooth PlayStation 3 (PS3) controller as a remote control interface to the robot for guiding the robot through each part of the experimental procedure (described in Section 3). The decisions and actions taken by the robot during the experiment were completely autonomous, but the timing of the actions was controlled by the press of a "next" button. The experimenter also used the controller to record distance measurements during the experiment and to provide ground truth information to the robot as to what the participant was communicating. The robot autonomously determined how to respond, based on the experimental conditions described in Section 4 .

Four small boxes were placed in the room, located at 0.75 meters and 1.5 meters from the centerline on each side (left and right) of the participant (Fig. 2). During the experiment (described in Section 3), the participant instructed the robot to look at those boxes. Each box was labeled with a unique shape and color; in this experiment, the shapes and colors matched the buttons on the PS3 controller: a green triangle, a red circle, a blue cross, and a purple square. This allowed the experimenter to easily indicate to the robot to which box the user was attending (i.e., ground truth).

A laser rangefinder on the robot was used to measure the distance from the robot to the participant's legs at all times. These measures were recorded for analysis purposes and were also used to inform robot behaviors, such as robot base movement and eye gaze (described below in Section 2.2).

The experimental setup is illustrated in Fig. 2 The experimental procedures within this setup are described in Section 3 The next section describes the robot's autonomous social behaviors.

\subsection{Robot Behaviors}

The robot executed three core social behaviors throughout the experiment: 1) forward and backward base movement, 2) maintaining eye contact with the participant, and 3) responding to participant's instructions with head movements and audio cues. Each behavior is described below.

Robot base movement was along a straight-line path directly in front of the participant and was limited to distances of 0.25 meters (referred to as the "near home" location) and 4.75 meters (referred to as the "far home" location); the robot returned repeatedly to these two "home" locations throughout the experiment. Robot velocity was proportional to the distance to the goal location; the maximum robot speed was $0.3 \mathrm{~m} / \mathrm{s}$, based on what has been shown to be acceptable (Satake et al. 2009).

As the robot moved, it maintained eye contact with the participant. The Bandit robot has eyes, but they are not actuated; to compensate, the robot pitched its head up or down, depending on the location of the participant's head, as determined by the distance to the participant (using the onboard laser) and the participant's self-reported height. Prolonged eye contact from the robot has been shown to increase user preferences of distance in HRI (Mumm \& Mutlu. 2011; Takayama \& Pantofaru, 2009). This was not a problem in the experiment, as the study is concerned with evaluating changes in user proxemic preferences rather than absolute preference values.

The robot provided head movement and audio cues to indicate whether or not it understood instructions provided by the participant (described in Section 3.3). If the robot understood the instructions, it provided an affirmative response (looking at a box); if the robot did not understand the instructions, it provided a negative response (shaking its head). With each head movement, one of two possible affective sounds was played to supplement the robot's response; affective sounds were used instead of speech, because robot speech influences proxemic preferences and would have introduced a confound variable in the experiment (Walters, Syrdal, Koay, Dautenhahn, \& te Boekhorst. 2008).

Using this setup, an experiment was performed to investigate user perceptions of robot performance attenuated by distance and its effect on user proxemic preferences. 


\section{Experimental Procedure and Measures}

The experimental procedure was performed in six sequential phases that included a human-robot interaction with behavioral and subjective measures. Fig. 3 illustrates the phases and includes the measures clarifying the order of events. Each phase is discussed below.

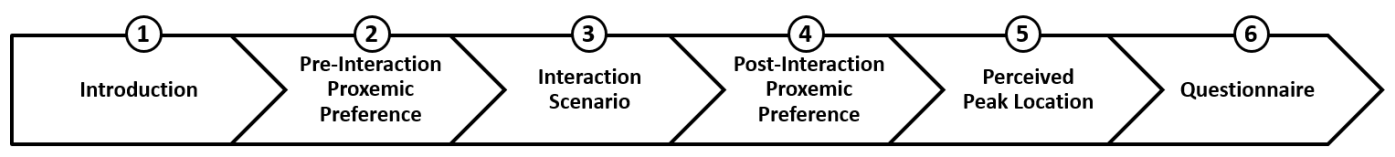

Figure 3. The six phases of the experimental procedure.

\subsection{Phase 1: Introduction}

Participants (described in Section 5) were greeted by the experimenter at the door of the private experimental space. They were informed of and agreed to the nature of the experiment and their rights, which included a statement that the experiment could be halted at any time.

Participants were then instructed by the experimenter to stand on a mark on the floor (denoted by the " $\mathrm{H}$ " in Fig. 2) and were asked to remain there for the duration of the experiment. The experimenter then provided instructions about the task to be performed.

Participants were introduced by the experimenter to the co-located robot and informed that all of its actions were completely autonomous. Participants were told that the robot would be moving along a straight line in the shared experimental space throughout the duration of the experiment. A brief demonstration of robot motion was provided. Participants were told that they would be asked about their preferences regarding the robot's location throughout the experiment.

Participants were then informed by the experimenter that they would be instructing the robot to look at any one of four boxes of their choosing located in the room (Fig. 2), and that they could use speech (in English) and pointing gestures. A vocabulary for robot instructions was provided: for speech, participants were told they could say the words "look at" followed by the name of the shape or color of each box (e.g., "triangle", "circle", "blue", "purple", etc.); for pointing gestures, participants were asked to use their left arm to point to boxes located on their left and their right arm to point to boxes on their right. This vocabulary was provided to minimize any participant perceptions that the robot did not understand their words or gestures; thus, use of the vocabulary attempted to maximize the perception that any failures of the robot were due to other factors.

Participants were told by the experimenter that they would repeat this instruction procedure to the robot many times, and that the robot would indicate whether or not it understood their instructions each time by using the head movements and audio cues, as described in Section 2.2 .

Participants had an opportunity to ask the experimenter any clarifying questions. The experiment commenced once participant understanding was verified.

\subsection{Phase 2: Pre-Interaction Proxemic Preference (pre)}

The robot autonomously moved to the "far home" location (Fig. 2). Participants were told by the experimenter that the robot would be approaching them and to say out loud the word "stop" when the robot reached the ideal location for a face-to-face conversation 5 This pre-interaction proxemic preference from the "far home" location is denoted by pre $_{\text {far }}$.

${ }^{5}$ Related work in human-robot proxemics asked participants about locations at which they felt comfortable Takayama \& Pantofaru 2009], yielding proxemic preferences very near to the participants. This work focuses on face-to-face human-robot \begin{tabular}{|l|l|l|l|l|}
\hline conversational interaction, with proxemic preference farther from the participants (Mead \& Matarić & 2016 & Torta et al. 2013 \\
\hline
\end{tabular} Torta, Cuijpers, Juola, \& van der Pol 2011, hence the choice of wording. 
When the participant was ready, the experimenter pressed a PS3 button to start the robot moving. When the participant said "stop," the experimenter pressed another button to halt robot movement. The experimenter pressed another button to record the distance between the robot and the participant, as measured by the on-board laser.

Once the pre $_{\text {far }}$ distance was recorded, the experimenter pressed another button, and the robot autonomously moved to the "near home" location (Fig. 22); the participant was informed that the robot would be approaching this location and would stop on its own. The process was repeated with the robot backing away from the participant, and the participant saying "stop" when it reached the ideal location for conversation. This pre-interaction proxemic preference from the "near home" location is denoted by pre $_{\text {near }}$.

Values for pre $_{\text {far }}$ and pre $_{\text {near }}$ were used to calculate and record the average pre-interaction proxemic preference, denoted by $p r \epsilon^{6}$

\subsection{Phase 3: Interaction Scenario}

After determining the participant's pre-interaction proxemic preferences, the robot autonomously returned to the "far home" location. The experimenter then repeated the instructions to the participant about the task they were to perform with the robot. When the participant verified understanding the task and indicated readiness, the experimenter pressed a button to proceed with the task.

The robot autonomously visited ten pre-determined locations along the line (Fig. 2). At each location, the robot responded to instructions from the participant to look at one of four boxes located in the room (Fig. 2). Five back-and-forth instruction-response interactions were performed at each location, after which the robot moved to the next location along its path; thus, each participant experienced a total of 50 instruction-response interactions. Robot goal locations were in 0.5 -meter intervals between the "near home" location ( 0.25 meters) and "far home" location ( 4.75 meters), inclusively, along a straight-line path in front of the participant (Fig. 2). Locations were visited in sequential order; to reduce ordering effects, for half of the participants, the robot approached from the "far home" location (i.e., farthest-to-nearest order), and for the other half of the participants, the robot backed away from the "near home" location (i.e., nearest-to-farthest order) (Murata, 1999).

To simulate social signal attenuation at each location in a controlled fashion, robot performance was artificially manipulated as a function of distance to the participant, as described in detail in Section 4. After each instruction provided by the participant, the experimenter provided to the robot (via a remote control interface) the ground truth of the instruction; the robot then determined whether or not it would have understood the instruction based on a prediction from a performance versus distance curve (specified by the assigned experimental condition described in Section 4). The robot then provided either an affirmative response or a negative response to the participant, indicating its successful or failed understanding of the instruction, respectively. Participants were not told how to react to robot responses, and each instruction-response pair was treated as independent.

The complete interaction session lasted 10-15 minutes, depending on participant speed.

\subsection{Phase 4: Post-Interaction Proxemic Preference (post)}

After the robot visited each of the ten locations, it returned to the "far home" location. The experimenter then repeated the procedure for determining proxemic preferences described in Section 3.2 This process generated post-interaction proxemic preferences from the "far home" and "near home" locations, as well as their average, denoted by post far, post $_{\text {near }}$, and pos 7 , respectively.

\footnotetext{
${ }^{6}$ Post hoc analysis revealed no statistically significant difference between pre $_{f a r}$ and pre $_{\text {near }}$ measurements, hence the use of pre.

${ }^{7}$ Post hoc analysis revealed no statistically significant difference between post $_{f a r}$ and post $_{\text {near }}$ measurements, hence the use of post.
} 


\subsection{Phase 5: Perceived Peak Location (perc)}

Finally, after collecting post-interaction proxemic preferences, the experimenter repeated the procedure described in Section 3.2, this time to determine participant perceptions of the location of peak performance. This process generated perceived peak performance locations from the "far home" and "near home" locations, as well as their average, denoted $\operatorname{perc}_{\text {far }}, \operatorname{perc}_{\text {near }}$, and per ${ }^{8}$, respectively. This final measurement marked the conclusion of the experiment.

\subsection{Phase 6: Questionnaire}

After the experiment concluded, participants were asked by the experimenter to complete a questionnaire about their experience with the robot. The multi-item questionnair ${ }^{9}$ measured subjective participant perceptions of the robot in terms of the Godspeed questionnaire metrics (Bartneck, Croft, Kulic, \& Zoghbi 2009)—animacy (9 items; Cronbach's $\alpha=0.88$ ), anthropomorphism (6 items; Cronbach's $\alpha=0.82$ ), competence (6 items; Cronbach's $\alpha=0.84$ ), likability (6 items; Cronbach's $\alpha=0.85$ ), and safety (4 items; Cronbach's $\alpha=0.55$ ) —as well as custom metrics of engagement (6 items; Cronbach's $\alpha=0.76$ ), comfort (6 items; Cronbach's $\alpha=0.72$ ), and technology adoption (6 items; Cronbach's $\alpha=0.65$ ). Participants responded to items for each multi-item measure on a seven-point Likert scale; items were randomly ordered prior to the study to reduce effects between them and were consistent across participants.

\section{Experimental Conditions}

During each interaction scenario (described in Section 3.3), robot performance-denoted by $p(x)$ was artificially varied based on distance $x$ in a between-participants design. Performance values for $p(x)$ were calculated a priori for each interaction and were approximately proportional to a scaled Gaussian distribution parameterized by the following values:

1. $\mu_{x}$, the maximum performance distance - the mean (center) of the distribution, treated as the actual location of robot peak performance;

2. $p_{\max }$, the maximum performance value - the maximum likelihood of recognizing a user instruction;

3. $p_{\min }$, the minimum performance value - the minimum likelihood of recognizing a user instruction; and

4. $p_{\text {avg }}$, the average performance value - the average likelihood of recognizing a user instruction, indicating how many affirmative and negative responses the robot produced during the interaction (e.g., if $p_{\text {avg }}=0.40$, then the robot provided 20 affirmative responses and 30 negative responses distributed across 50 instructions).

The first three of these parameters- $\mu_{x}, p_{\max }$, and $p_{\min }$-were systematically varied in between-participants conditions, as discussed in Sections 4.1 4.3, these manipulations influenced the fourth parameter $p_{a v g}$, which in turn impacted the way that robot responses were distributed across locations between conditions (Table 11). In addition to the three parameter-varying conditions, a uniform distribution of $p(x)$ was also evaluated to serve as a baseline (BL) betweenparticipants condition (discussed in Section 4.4.

\footnotetext{
${ }^{8}$ Post hoc analysis revealed no statistically significant difference between $\operatorname{per} c_{f a r}$ and perc $_{n e a r}$ measurements, hence the use of perc.

${ }^{9}$ Cronbach's $\alpha$ is reported for each multi-item measure to indicate internal consistency between items in the measure; $\alpha \geq 0.7$ is commonly considered acceptable.
} 
For all conditions, the performance value $p\left(\mu_{x}\right)$ at $\mu_{x}$ was always $p_{\max }$ (i.e., the number of affirmative responses was 5 for $p_{\max }=1.00$ ), and the number of affirmative responses at other distances was always less than that at $\mu_{x}$, to ensure that participants were exposed to an actual singular maximum performance value.

The maximum performance distance was held constant in conditions that varied the maximum and minimum performance values ( $p_{\max }$ and $p_{\min }$, respectively). In these conditions, a constant maximum performance distance of $\mu_{x}=2.25$ was chosen for three reasons:

1. It was the near-center location between all locations explored, allowing for a balance in the number of affirmative robot responses on each side of the location of maximum performance.

2. It was at a location that is not where people initially preferred the robot to be, based on Mead and Matarić (2015), which reported user proxemic (distance) preferences of $M=1.14, S D=$ 0.49 meters to the same robot as was used in this study.

3. It was at a location predicted to have both acceptable and functional combined performance of actual automated speech and gesture recognition systems (Mead \& Matarić, 2016).

An overview of the values for the baseline and parameter-varying conditions-varying $\mu_{x}$, $p_{\max }$, and $p_{\min }$ (and subsequently, $p_{\text {avg }}$ ) —as well as the corresponding distribution of affirmative responses in each condition is presented in Table 1 In each condition, the number of affirmative responses was normalized to a desired average robot performance value $p_{\text {avg }}$. The order of the five possible robot responses was randomized at each location.

Each of the four experimental conditions is detailed in corresponding sections below.

Table 1: The distribution of hard-coded affirmative responses provided by the robot across baseline (BL) and parameter-varying conditions; note that average performance values $p_{\text {avg }}$ vary as well. Manipulated values in each condition are highlighted in bold italics. Each table row represents a complete experimental condition to which a participant was exposed.

\begin{tabular}{|c|c|c|c|c|c|c|c|c|c|c|c|c|c|c|c|}
\hline & \multicolumn{4}{|c|}{ Performance Parameters } & \multicolumn{10}{|c|}{ Distance, $x$ (meters) } \\
\hline & & $\mu_{x}$ & $p_{\max }$ & $p_{\min }$ & $p_{\text {avg }}$ & \begin{tabular}{|l|l|}
0.25 \\
\end{tabular} & 0.75 & \begin{tabular}{|l|}
1.25 \\
\end{tabular} & 1.75 & 2.25 & \begin{tabular}{|l|}
2.75 \\
\end{tabular} & 3.25 & 3.75 & 4.25 & 4.75 \\
\hline \multirow{9}{*}{ 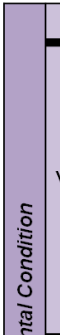 } & $\mathrm{BL}$ & N/A & 0.40 & 0.40 & 0.40 & 2 & 2 & 2 & 2 & 2 & 2 & 2 & 2 & 2 & 2 \\
\hline & \multirow{8}{*}{$\begin{array}{c}\text { Vary } \\
\mu_{x}\end{array}$} & 0.75 & 1.00 & 0.00 & 0.40 & 4 & 5 & 4 & 3 & 1 & 1 & 1 & 1 & 0 & 0 \\
\hline & & 1.25 & 1.00 & 0.00 & 0.40 & 3 & 4 & 5 & 4 & 3 & 1 & 0 & 0 & 0 & 0 \\
\hline & & 1.75 & 1.00 & 0.00 & 0.40 & 1 & 3 & 4 & 5 & 4 & 3 & 0 & 0 & 0 & 0 \\
\hline & & 2.25 & 1.00 & 0.00 & 0.40 & 0 & 1 & 3 & 4 & 5 & 4 & 3 & 0 & 0 & 0 \\
\hline & & 2.75 & 1.00 & 0.00 & 0.40 & 0 & 0 & 0 & 3 & 4 & 5 & 4 & 3 & 1 & 0 \\
\hline & & \begin{tabular}{|l}
3.25 \\
\end{tabular} & 1.00 & 0.00 & 0.40 & 0 & 0 & 0 & 0 & 3 & 4 & 5 & 4 & 3 & 1 \\
\hline & & 3.75 & 1.00 & 0.00 & 0.40 & 0 & 0 & 0 & 0 & 1 & 3 & 4 & 5 & 4 & 3 \\
\hline & & 4.25 & 1.00 & 0.00 & 0.40 & 0 & 0 & 1 & 1 & 1 & 1 & 3 & 4 & 5 & 4 \\
\hline & \multirow{4}{*}{$\begin{array}{l}\text { Vary } \\
p_{\max }\end{array}$} & 2.25 & 0.20 & 0.00 & 0.02 & 0 & 0 & 0 & 0 & 1 & 0 & 0 & 0 & 0 & 0 \\
\hline & & 2.25 & 0.40 & 0.00 & 0.20 & 1 & 1 & 1 & 1 & 2 & 1 & 1 & 1 & 1 & 0 \\
\hline & & 2.25 & 0.60 & 0.00 & 0.30 & 1 & 1 & 2 & 2 & 3 & 2 & 2 & 1 & 1 & 0 \\
\hline & & 2.25 & 0.80 & 0.00 & 0.40 & 1 & 1 & 3 & 3 & 4 & 3 & 3 & 1 & 1 & 0 \\
\hline & \multirow{4}{*}{$\begin{array}{l}\text { Vary } \\
p_{\text {min }}\end{array}$} & 2.25 & 1.00 & 0.20 & 0.50 & 1 & 1 & 3 & 4 & 5 & 4 & 3 & 2 & 1 & 1 \\
\hline & & 2.25 & 1.00 & 0.40 & 0.60 & 2 & 2 & 3 & 4 & 5 & 4 & 3 & 3 & 2 & 2 \\
\hline & & 2.25 & 1.00 & 0.60 & 0.70 & 3 & 3 & 3 & 4 & 5 & 4 & 4 & 3 & 3 & 3 \\
\hline & & 2.25 & 1.00 & 0.80 & 0.82 & 4 & 4 & 4 & 4 & 5 & 4 & 4 & 4 & 4 & 4 \\
\hline & & & & & & \multicolumn{10}{|c|}{ \# Affirmative Responses vs. Distance } \\
\hline
\end{tabular}




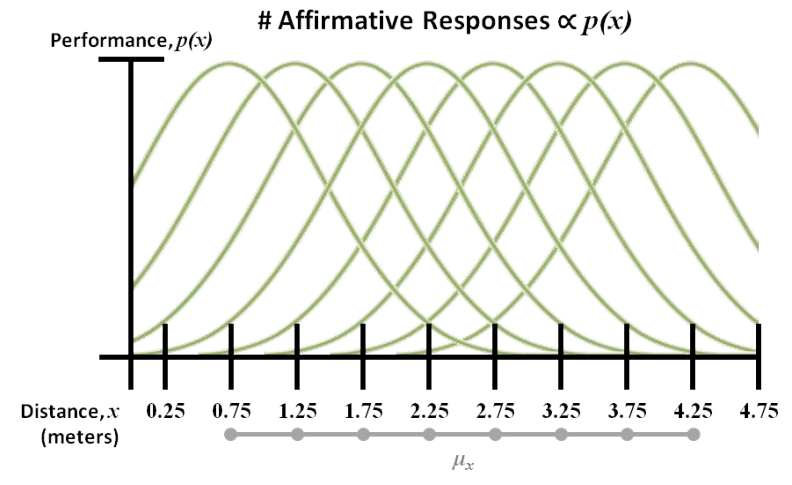

Figure 4. Each curve represents an experimental manipulation condition (to which a participant was exposed) varying the maximum performance distance, $\mu_{x}=\{0.75,1.25,1.75,2.25,2.75,3.25,3.75,4.25\}$ meters.

\subsection{Condition: Maximum Performance Distance $\left(\mu_{x}\right)$}

To explore the space of human responses to robot performance differences at a variety of distances, the maximum performance distance $\mu_{x}$ was varied by selecting the eight locations non-inclusively between the "near home" and "far home" locations (Figs. 22and47; the "near home" and "far home" locations were not included in the set of $\mu_{x}$ to ensure that participants were always exposed to an actual peak in performance, rather than to just a trend. The value of $\mu_{x}$ was varied between participants. The number of affirmative responses was normalized to $20(40 \%)$ to ensure a consistent user experience of average robot performance $p_{\text {avg }}=0.40$ for different values of $\mu_{x}$.

\subsection{Condition: Maximum Performance Value $\left(p_{\max }\right)$}

To investigate how participants responded to differences in robot maximum performance, $p_{\max }$ was varied at a constant location of maximum performance, $\mu_{x}=2.25$. Values of $p_{\max }$ were $0.20,0.40$, 0.60 , and 0.80 (Fig. 5); for analysis, $p_{\max }=1.00$ at $u_{x}=2.25\left(p_{\text {avg }}=0.40\right)$ collected in the condition varying $\mu_{x}$ (Section 4.1) was also considered as part of this group. Average performance values naturally varied in this condition, with $p_{\text {avg }}=\{0.02,0.20,0.30,0.40\}$.

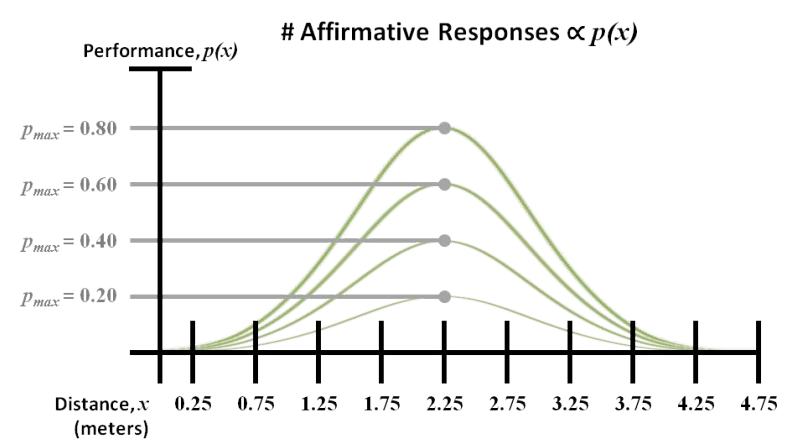

Figure 5. Each curve represents an experimental manipulation condition (to which a participant was exposed) varying the maximum performance value, $p_{\max }=\{0.20,0.40,0.60,0.80\}$. 


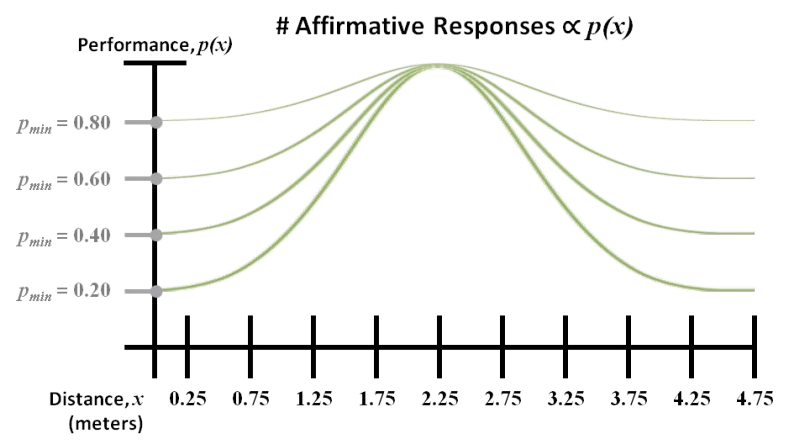

Figure 6. Each curve represents an experimental manipulation condition (to which a participant was exposed) varying the minimum performance value, $p_{\min }=\{0.20,0.40,0.60,0.80\}$.

\subsection{Condition: Minimum Performance Value $\left(p_{\min }\right)$}

The experiment also investigated how people responded to differences in robot minimum performance. In this condition, $p_{\min }$ was varied in a way similar to that of $p_{\max }$ (Section 4.2), with $\mu_{x}=2.25$. Values of $p_{\min }$ were $0.20,0.40,0.60$, and 0.80 ; for analysis, $p_{\min }=0.00$ at $u_{x}=2.25\left(p_{a v g}=0.40\right)$ collected in the condition varying $\mu_{x}$ (Section 4.1) was also considered as part of this group. Average performance values naturally varied in this condition, with $p_{\text {avg }}=\{0.50,0.60,0.70,0.82\}$.

\subsection{Condition: Baseline $\left(p(x)=p_{\max }=p_{\min }\right)$}

In this condition, robot performance was the same at all locations (Fig. 7 and Table 11); in this work, $p_{\max }=p_{\min }=0.40$, as this is an average performance rate predicted by our previous work for traditional human-robot proxemic configurations (Mead \& Matarić, 2016). Thus, at each of the ten locations visited, the robot provided two affirmative and three negative responses, respectively. This condition served as a baseline of participant proxemic preferences within the task.

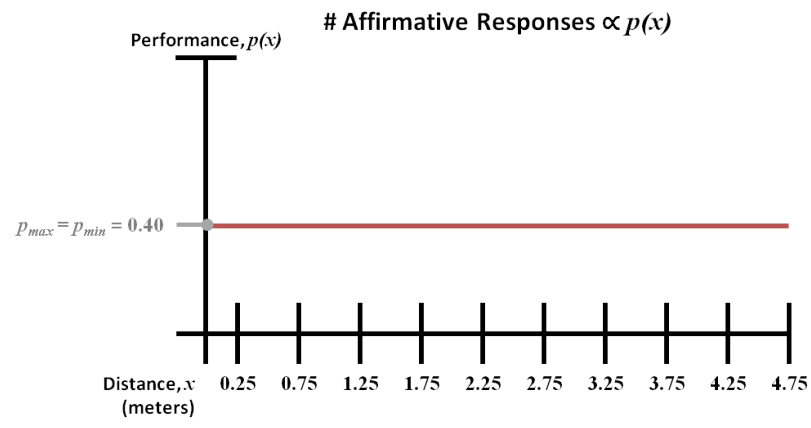

Figure 7. The line represents the experimental baseline condition (to which a participant was exposed), where performance versus distance is represented by a uniform distribution $p(x)=p_{\max }=p_{\min }=0.40$. 


\section{Experimental Hypotheses and Participants}

Within these conditions, three hypotheses were tested with respect to pre-interaction location (pre), post-interaction location (post), and perceived peak location (perc) behavioral measures:

H1: In the baseline condition, there will be no change in participant proxemic preferences.

H2: In the $\mu_{\mathrm{x}}$-varying condition, participants will be able to identify a relationship between robot performance and human-robot proxemics.

H3: In the $\mu_{\mathrm{x}}$-varying condition, participants will adapt their proxemic preferences to improve robot performance.

The evaluation of each hypothesis is discussed in the corresponding Sections 6.1.1 6.1.3 below.

To test these hypotheses, we recruited 180 participants (90 male, 90 female) from the campus community at the University of Southern California. Participant race was diverse (105 white/Caucasian, 57 Asian, 8 Latino/Latina, 7 black/African-American, and 3 mixed-race). All participants reported proficiency in English and had lived in the United States for at least two years (i.e., had acclimated to U.S. culture). The average age (in years) of participants was 21.34 ( $S D=4.26)$, ranging from 18 to 39 . On a seven-point scale, participants reported moderate familiarity with technology $(M=4.04, S D=0.93)$. The average participant height (in meters) was $1.72(S D=0.14)$, ranging from 1.49 to 1.96 . Related work reports how human-robot proxemics is influenced by participant gender and technology familiarity (Takayama \& Pantofaru. 2009), culture (Eresha, Haring, Endrass, Andre, \& Obaid 2013), and height (Hiroi \& Ito, 2011, Rae, Takayama, \& Mutlu. 2005).

The 180 participants were randomly assigned to a condition, with $N_{B L}=20$ in the baseline (BL) condition, and $N_{\mu_{x}}=80, N_{p_{\max }}=40$, and $N_{p_{\min }}=40$ in the conditions that varied maximum performance distance $\left(\mu_{x}\right)$, maximum performance value $\left(p_{\max }\right)$, and minimum performance value $\left(p_{\min }\right)$, respectively. Within each parameter-varying condition, the participants were randomly assigned to one of the subconditions (e.g., one of the eight maximum performance distances described in Section 4 with $N=10$ for each subcondition. Neither the participant nor the experimenter was aware of the assigned condition.

\section{Experimental Results}

The analyses and results of the experiment were computed relative to both behavioral measures (Section 6.1) and subjective measures (Section 6.2). Implications of these results for autonomous sociable robots and human-robot proxemics are discussed in Section 7

To provide a baseline of the robot used for comparison in general human-robot proxemics, preinteraction proxemic preferences (pre) across all conditions $(N=180)$ were consolidated and analyzed, as the data had not yet been influenced by the manipulation in the assigned experimental condition. The participant pre-interaction proxemic preference (in meters) was determined to be $(M=1.14, S D=0.49)$ meters for the Bandit robot system, which is consistent with Mumm and Mutlu (2011) and Mead and Matarić (2016) but twice as far as related work has reported for robots of a similar form factor (Takayama \& Pantofaru, 2009: Walters et al., 2009).

\subsection{Analysis of Behavioral Measures}

6.1.1 H1: Pre-Versus Post-interaction Locations. To test H1 (there will be no change in proxemic preferences in the baseline conditions), pre-interaction proxemic preferences ( $p r e$ ) were compared to post-interaction proxemic preferences (post) of participants in the baseline condition.

A paired $t$-test revealed a statistically significant change in participant proxemic preferences between pre $(M=1.12, S D=0.51)$ and post $(M=1.39, S D=0.63) ; t(39)=1.49, p=0.02$ (Fig. 8). Thus, hypothesis $\mathbf{H 1}$ was not supported. 


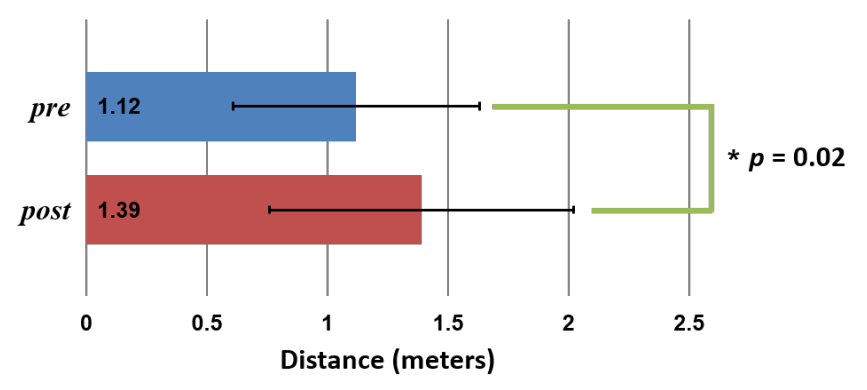

Figure 8. Participant proxemic preferences; pre-interaction (pre) versus postinteraction (post).

This result suggests that the context of the interaction scenario itself influenced participant proxemic preferences. To address any influence the scenario might have on subsequent analyses, a contextual offse $t^{10} \theta$ was defined as the average difference in participant post-interaction and pre-interaction proxemic preferences $(M=0.27, S D=0.48)$; this $\theta$ value is subtracted from (post - pre) values in Section 6.1.3 to normalize for the interaction context.

6.1.2 H2: Perceived Versus Actual Peak Locations. To test $\mathbf{H 2}$ (participants will identify a relationship between robot performance and human-robot proxemics), participant-perceived locations of peak performance (perc) were compared to actual locations of peak performance $\left(\right.$ peak $\left.=\mu_{x}\right)$ in the $\mu_{x}$-varying conditions (Fig. 9).

Steven's power law, $a x^{b}$, has previously been used to model human distance estimation as a function of actual distance (Murata, 1999) and is generally representative of human perceived versus actual stimuli (Stevens, 2007). However, existing power laws relevant to this work only pertain to distances of 3-23 meters, which are beyond the range of the natural face-to-face communication this work focuses on. Thus, the goal here is to model the data to establish a power law for perc versus peak at locations more relevant to HRI (0.75-4.25 meters), which can then be evaluated to test $\mathbf{H 2}$.

Observations of the dataset indicated that the data were heteroscedasti ${ }^{11}$ (Fig. 9) —in this case, the variance appears to increase with distance from the participant, meaning that traditional statistical tests could not be applied. The Breusch-Pagan test for non-constant variance (NCV) confirmed this; $\chi^{2}(1, N=80)=15.79, p<0.001$. A commonly used and accepted approach to alleviate heteroscedasticity is to transform the perc and peak data to a log-log scale. While not applicable to all datasets, this approach served as an adequate approximation for the collected data (Fig. 10); it also supports the application of a regression analysis to determine parameter values for the power law coefficient and exponent, $a=1.32$ and $b=0.51$, respectively. With these parameters, it was revealed that peak was a strongly correlated and very significant predictor of perc; $R^{2}=0.50, F(1,78)=76.48, p<0.001$. Thus, hypothesis $\mathbf{H 2}$ was supported.

This result suggests that people are able to identify a relationship between robot performance and human-robot proxemics, but they are predictably poor at estimating the distance, $x$, to the location of peak performance, based on the power law equation $1.32 x^{0.51}$. While human estimation of the location of peak performance is suboptimal, it is possible that repeated exposure to the robot over multiple sessions might yield more accurate results.

\footnotetext{
${ }^{10}$ We use the term "contextual" to indicate that this offset is constrained to a particular scenario (i.e., the experiment).

11 "Heteroscedasticity" implies that the variance of the dependent variable is a function of the predictor variable.
} 


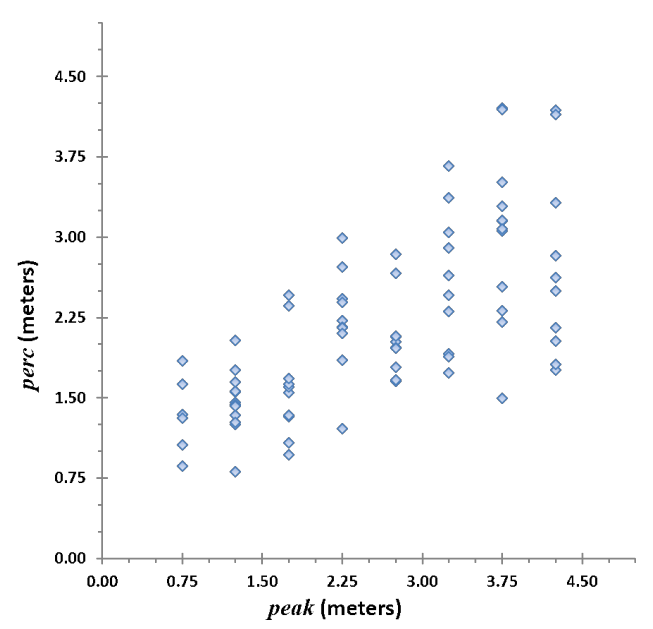

Figure 9. Participant-perceived location of robot peak performance (perc) versus actual location of robot peak performance $(p e a k)$. Note the heteroscedasticity of the data, which prevents the use of traditional statistical analyses without first transforming the data (shown in Fig. 10p.

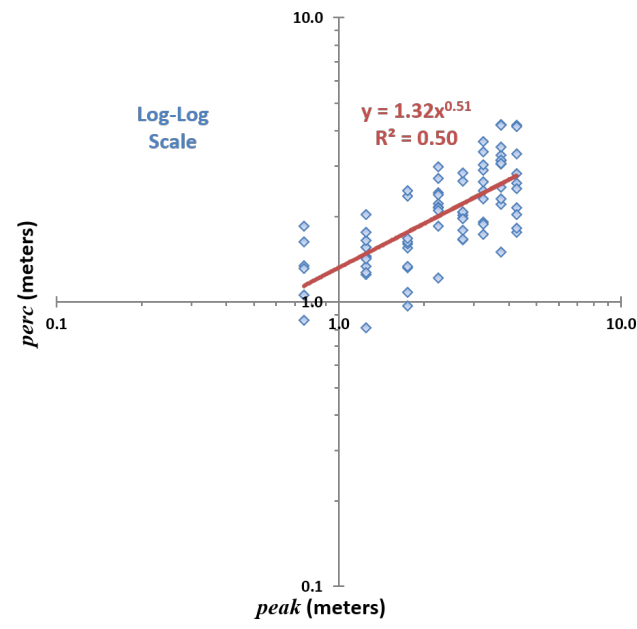

Figure 10. Participant-perceived location of robot peak performance (perc) versus actual location of robot peak performance (peak) on a log-log scale, reducing the effects of heteroscedasticity and allowing us to perform regression to determine parameters of the power law, $a x^{b}$.

6.1.3 H3: Preferences Versus Peak Locations. To test $\mathbf{H 3}$ (participants will adapt their proxemic preferences to improve robot performance), changes in participant pre-/post-interaction proxemic preferences (post - pre $-\theta$ ) were compared to the distance from the participant pre-interaction proxemic preference to either a) the actual location of robot peak performance (peak-pre) (Fig. 11), or b) the perceived location of robot peak performance (perc-pre) (Fig. 12), both in the $\mu_{x}$-varying conditions. 
Data for (post - pre - $\theta$ ) versus both (peak - pre) and (perc - pre) were heteroscedastic, as indicated by Breusch-Pagan NCV tests: $\chi^{2}(1, N=80)=18.81, p<0.001$; and $\chi^{2}(1, N=80)=$ $13.55, p<0.001$; respectively. This is intuitive, as the data for perceived (perc) versus actual (peak) locations of peak performance were also heteroscedastic (Fig. 9p. The log-transformation approach used in Section 6.1.2 did not perform well in modeling these data; thus, an alternative approach was needed. A Generalized Linear Model (GLM) (Nelder \& Wedderburn, 1972) was utilized because it models the variance of each measurement separately as a function of predicted values and allows appropriate statistical tests for significance to be applied.

Changes were modeled in participant proxemic preferences (post - pre $-\theta$ ) versus distance from pre-interaction proxemic preference to the actual (rather than perceived) location of peak performance (peak - pre). In the ideal situation (for the robot), these match almost one to one-in other words, the participant meets the needs of the robot entirely by changing proxemic preferences to be centered at the peak of robot performance. Unfortunately for the robot, this was not the case. A strongly correlated and statistically significant relationship was detected between participant proxemic preference change and distance from pre-interaction preference to the peak location $\left(R^{2}=0.55, \beta=0.54, t[78]=9.71, p<0.001\right)$, but participant preference change was predictably poor $(\beta=0.54)$ with respect to robot location of peak performance (Fig. 111).

Recall that results reported for $\mathbf{H 2}$ (Section 6.1.2) suggested that, while people do perceive a relationship between robot performance and distance, their ability to accurately identify the location of robot peak performance diminishes based on the distance to it, as predicted by a power law. Were participants trying to maximize robot performance but simply adapting their preferences to a suboptimal location?

This question was investigated by considering changes in participant proxemic preferences (post - pre $-\theta$ ) versus distance from pre-interaction proxemic preference to the perceived (rather than actual) location of peak performance (perc - pre). If the participant was adapting their proxemic preferences to accommodate the needs of the robot, then these should match almost one to one. A GLM was fit to these data and yielded a strongly correlated and statistically significant relationship between changes in proxemic preferences and perceptions of robot performance $\left(R^{2}=0.54, \beta=0.93, t[78]=9.61, p<0.001\right)$ (Fig. 12). Thus, hypothesis $\mathbf{H 3}$ was supported.

The near perfect correspondence $(\beta=0.93)$ between post-interaction proxemic preferences and participant perceptions of robot peak performance is compelling, suggesting that participants adapted their proxemic preferences almost entirely to improve robot performance in the interaction.

\subsection{Analysis of Subjective Measures}

Bidirectional stepwise multivariate linear regression was used to model and analyze the relationships between participant subjective experiences of the robot (described in Section 3.6) and the four parameters of the distribution of robot responses (i.e., $\mu_{x}, p_{\max }, p_{\min }$, and $p_{\text {avg }}$; described in Section (4) across all conditions. Of the eight subjective factors considered, significant effects were found for five of them-competence, anthropomorphism, engagement, likability, and technology adoption. Fig. 13 illustrates the correlation coefficient $(\beta)$ and statistical significance $(p)$ of each predictor-measure pair represented in the resulting regression models. For these analyses, $t$-tests determined statistical significance $(p)$, and adjusted- $R^{2}$ values $\left(R_{A}^{2}\right)$ indicate the explanatory power of the model based on the number of predictors. A detailed summary of the results is provided below and discussed further in Section 7.2 .

6.2.1 Subjective Measure: Competence. Values of average performance $\left(p_{\text {avg }}\right)$ and minimum performance $\left(p_{\min }\right)$ were found to be significant predictors of perceived competence $\left(R_{A}^{2}=0.43, \beta_{0}=\right.$ $1.35)$, with $(\beta=2.80, p=0.01)$ and $(\beta=1.78, p=0.04)$, respectively; furthermore, maximum 


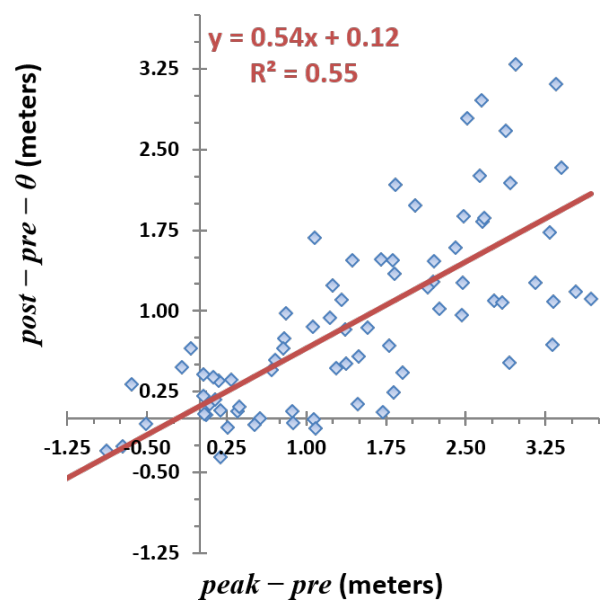

Figure 11. Changes in participant pre-/post-interaction proxemic preferences (pre and post, respectively; $\theta$ is the contextual offset defined in Section 6.1.1) versus distance from participant pre-interaction proxemic preference (pre) to the actual location of robot peak performance (peak).

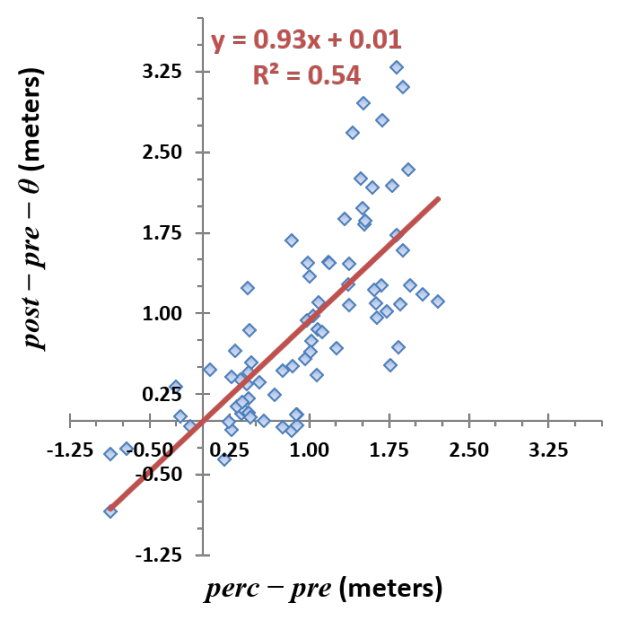

Figure 12. Changes in participant pre-/post-interaction proxemic preferences (pre and post, respectively; $\theta$ is the contextual offset defined in Section 6.1.1 versus distance from participant pre-interaction proxemic preference (pre) to the perceived location of robot peak performance (perc).

performance $\left(p_{\max }\right)$ was a marginal predictor of perceived competence $(\beta=0.86, p=0.09)$. This indicates that robot performance factors should be strongly considered when deploying a sociable robot, as the perceptions of intelligence attributed by a co-present human user will vary based on how well the robot functions. Average performance should be as high as possible, as it is the deciding factor in perceptions of intelligence. Situations in which the robot fails should be avoided, as they hinder perceived robot competence. High robot maximum performance should be a tertiary objective, as it is only marginally predictive of perceived intelligence. 


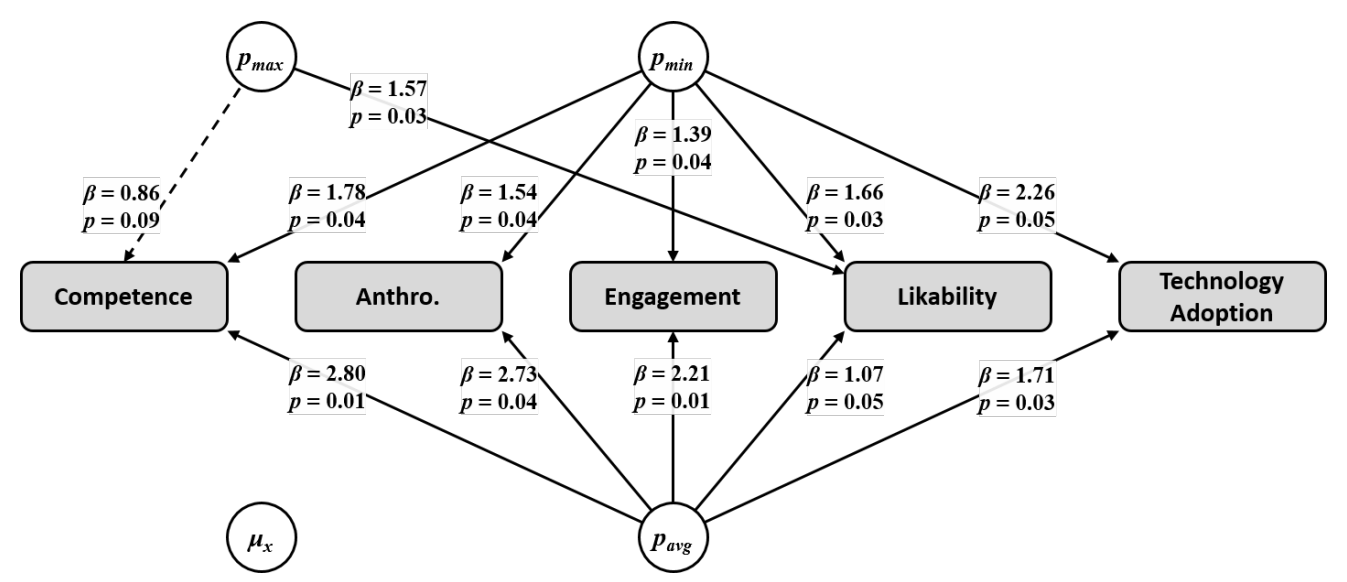

Figure 13. Significant relationships modeled between the manipulated predictor variables and subjective measures. The correlation coefficient $(\beta)$ and statistical significance $(p)$ for each predictor-measure pair is presented along the connecting line; a dotted line indicates marginal significance.

6.2.2 Subjective Measure: Anthropomorphism. Values of average performance $\left(p_{\text {avg }}\right)$ and minimum performance $\left(p_{\text {min }}\right)$ were found to be significant predictors of perceived anthropomorphism $\left(R_{A}^{2}=0.41, \beta_{0}=1.70\right)$, with $(\beta=2.73, p=0.04)$ and $(\beta=1.54, p=0.04)$, respectively. This suggests that a robot that is consistently performing well at many locations will receive the greatest attributions of agency and human-likeness; furthermore, reducing the occurrence of robot failure will increase these attributions.

6.2.3 Subjective Measure: Engagement. Values of average performance $\left(p_{\text {avg }}\right)$ and minimum performance $\left(p_{\min }\right)$ were found to be significant predictors of participant engagement $\left(R_{A}^{2}=\right.$ $\left.0.38, \beta_{0}=2.88\right)$, with $(\beta=2.21, p=0.01)$ and $(\beta=1.39, p=0.04)$, respectively. Thus, for increased user engagement, the robot should perform well on average and should avoid situations in which it would likely fail. In situations where the robot was consistently failing (i.e., when both average and minimum performance were low), it was clear from experimenter observations that participants were significantly less engaged than when the robot was performing better. Interestingly, experimenter observations of participants also suggested that participants appeared bored (i.e., less engaged) when minimum performance was at the highest level explored $\left(p_{\min }=0.80\right)$, as the robot performed very well at every location, though participant questionnaire responses did not support this observation. Related work has shown that people are more engaged by a robot that produces imperfect or unexpected behaviors (in Short, Hart, Vu, and Scassellati (2010), the robot cheated during a game of rock-paper-scissors), which might offer insights into this observation.

6.2.4 Subjective Measure: Likability. Values of minimum performance $\left(p_{\min }\right)$, maximum performance $\left(p_{\max }\right)$, and average performance $\left(p_{\text {avg }}\right)$ were found to be significant predictors of perceived likability $\left(R_{A}^{2}=0.32, \beta_{0}=1.63\right)$, with $(\beta=1.66, p=0.03),(\beta=1.57, p=0.03)$, and $(\beta=1.07, p=0.05)$, respectively. People do not like it when the robot consistently fails-this is intuitive but had not previously been well quantified in HRI. Increased average performance provided some support and could be a deciding factor in a user's liking of the robot. 
6.2.5 Subjective Measure: Technology Adoption. Values of minimum performance $\left(p_{\min }\right)$ and average performance $\left(p_{\text {avg }}\right)$ were found to be significant predictors of participant technology adoption $\left(R_{A}^{2}=0.32, \beta_{0}=2.40\right)$, with $(\beta=2.26, p=0.05)$ and $(\beta=1.71, p=0.03)$, respectively. Repetitive failures can dramatically reduce the possibility of a user adopting the technology, even if the robot performs well on average. Maximum performance is therefore not the most important factor in design — rather, avoidance of failure should be the objective.

\section{Discussion}

The results obtained from this study have significant implications for the design of sociable robots and autonomous robot proxemic control systems. Sections 7.1 and 7.2 discuss these implications with respect to behavioral and subjective results, respectively.

\subsection{Implications of Behavioral Results}

The primary implication of the behavioral results reported in Section 6.1 is that the proxemic preferences of human users will change over time as users interacts with and come to understand the needs of the robot. These changes will, in turn, improve robot performance (an objective measure of automated speech and gesture recognition rates).

As illustrated in our previous work, the locations of on-board sensors for social signal recognition (e.g., microphones and cameras), as well as the automated speech and gesture recognition methods used, can have significant impact on the performance of the robot in autonomous faceto-face social interactions (Mead \& Matarić, 2016). The behavioral results reported for this study suggest that people will adapt their behavior in an effort to improve robot performance, so it is anticipated that human-robot proxemics will vary between robot platforms with different hardware and software configurations based on factors that are:

1. not specific to the user-unlike culture (Eresha et al., 2013), gender, personality, or familiarity with technology (Takayama \& Pantofaru. 2009);

2. not observable by the user-unlike height (Hiroi \& Ito, 2011; Rae et al., 2005), amount of eye contact (Mumm \& Mutlu, 2011, Takayama \& Pantofaru, 2009), vocal parameters (Walters et al. 2008), or actual robot performance (as discussed in Section 6.1.2); and

3. not observable by the robot-the robot does not have ground-truth for its own performance but could predict its own recognition rates based on measurable properties of human social signals (e.g., voice loudness or gesture locations) (Mead \& Matarić, 2016).

User understanding of the relationship between robot performance and human-robot proxemics is a latent factor that only develops through repeated interactions with the robot (perhaps expedited by the robot communicating its predicted error); fortunately, the results presented here indicate that user understanding will develop in a predictable way. Thus, it is recommended that developers of sociable robots consider and perhaps model robot performance as a function of conditions that might occur in dynamic proxemic interactions with human users to predict and accommodate how the people will actually use the technology. This dynamic relationship will, in turn, enable richer autonomy for sociable robots by improving the performance of their automated recognition systems.

If developers adopt models of robot performance as a factor contributing to human-robot proxemics, then it follows that proxemic control systems should be designed to expedite the process of autonomously positioning the robot at an optimal distance from the user to maximize robot performance while still accommodating the initial personal space preferences of the user. This is the focus 
of our previous work, which treated proxemics as an optimization problem that considers the production and perception of social signals (speech and gesture) as a function of distance and orientation (Mead \& Matarić 2016). An objective of this study was to address questions regarding whether or not users would accept a robot that positions itself in locations that might differ from their initial proxemic preferences. The behavioral results (reported in Section 6.1.3) support the notion that user proxemic preferences will change through interactions with the robot as its performance is observed, and that the new user proxemic preference will be at the perceived location of robot peak performance. An extension of this result is that, through repeated interactions, user proxemic preferences will further adapt and eventually converge to the actual location of robot peak performance.

\subsection{Implications of Subjective Results}

The primary implication of the subjective results reported in Section 6.2 is that the relationship between robot performance and participant subjective experiences should be strongly considered by developers, if sociable robots are to be successfully deployed.

Overall, the subjective results of this study suggest that average performance across multiple interactions, rather than maximum performance in a select few interactions, should be a focus of robot developers, especially when considering human perceptions of robot competence, anthropomorphism, and engagement. Furthermore, in consideration of all reported significant effects, a robot developer should have the goal of minimizing the amount of user exposure to robot failure (as indicated by the effect of minimum performance values); if the robot can predict conditions in which it will most likely fail at recognizing user input (e.g., at certain physical locations, or based on the detected sound pressure level of speech or the regions of space occupied by gestures (Mead \& Matarić. 2016), then the robot could autonomously determine how to react in those situations. This issue is addressed by our previous work, wherein the robot selects proxemic behaviors that it predicts will maximize its ability to autonomously recognize human multimodal communication (speech and gesture), thus attempting to continuously optimize system performance throughout the duration of the interaction with a human user (Mead \& Matarić, 2016).

While no statistically significant relationship was identified between the location of maximum performance $\left(\mu_{x}\right)$ and participant subjective experiences along any measure, this serves as an important result. Previous work in human-robot proxemics predicts changes in factors such as perceived safety (Henkel, Murphy, \& Bethel, 2012), comfort/intimacy (Henkel et al. 2012, Mumm \& Mutlu. 2011; Takayama \& Pantofaru, 2009), and likability (Henkel et al., 2012; Mumm \& Mutlu. 2011); similar changes are also predicted in human-human proxemics literature (Argyle \& Dean, 1965. Hall, 1966). This could be explained by the factors that govern proxemic behavior and how people perceive them. The psychophysical representation of Hall (1963) suggests that human-human proxemic behavior can be represented by the sensory experiences of people at different distances, and that the four psychological proxemic zones (public, social, personal, and intimate) that encode the interpersonal relationship between two people are characterized by these sensory experiences (Hall. 1966). The implication then is that if certain sensory experiences are different or absent during a face-to-face interaction between two agents (human or robot), then perceptions of factors such as comfort or intimacy could not be predicted by distance alone-in short, sensory experience, rather than distance, is the significant variable in predicting these subjective measures (as suggested by the results reported in Section 6.2. This work adds further support for the representation of proxemic behavior reported in Mead, Atrash, and Matarić (2012), which proposed that the relationship between psychological and physical factors of proxemics (Mumm \& Mutlu, 2011) goes through a psychophysical layer of agent sensory experience; modeling social signal generation and recognition within this framework has been shown to objectively improve robot performance in autonomous HRI (Mead, Atrash, \& Matarić, 2013; Mead \& Matarić, 2016). 


\section{Limitations}

The experimental conditions were designed to be principled, rigorous, and extensive; however, there are limitations that should be considered, as follows.

The maximum and minimum performance values were varied in isolation (e.g., $p_{\max }=1.00$ when $p_{\min }$ was varied); however, in the real world, these recognition rates would likely vary together and in more complex ways (Mead \& Matarić, 2016) (e.g., if $p_{\max }=0.80$ and $p_{\min }=0.40$ ), which might result in interaction effects between factors. Additionally, participants were exposed to more examples of the manipulated variable when $p_{\min }$ was varied versus when $p_{\max }$ was varied; this choice was made to highlight the behavioral outcomes reported in Section 6.1 but might have reduced the effects of $p_{\max }$ on subjective outcomes. Thus, the results presented here simply provide evidence to the effects of robot performance on human perceptions and aim to serve as guidelines rather than rigid rules about subjective experiences in response to variability in robot performance.

We used coarse Gaussian approximations of robot automated recognition rates as a function of distance; however, our results from Mead and Matarić (2016) indicate that these distributions in the real world are by no means normal and are often multimodal (when recognition rates of multiple social signals are combined). This consideration introduces a new design space of human-robot proxemic behavior. The general research question asks, "How will people adapt their proxemic preferences in any given performance field in which performance varies with a variety of factors, such as distance, orientation, and environmental interference?" The follow-up question then asks "How can the robot expedite the process of establishing an appropriate human-robot proxemic configuration within the performance field without causing user discomfort?"

\section{Summary and Conclusions}

To be effective, an autonomous sociable robot must meet the needs and preferences of the human user (Feil-Seifer \& Matarić, 2005); however, as our study and results suggest, this should not be done at the expense of the robot's own ability to understand the user's social signals. In particular, human proxemic preferences with respect to a robot can have significant impact on the performance of the robot's automated speech and gesture recognition systems (Mead \& Matarić, 2016). Thus, for a successful interaction, robot performance requirements may be inconsistent with the preferences of the human user and might require changes in those preferences.

This work investigated how user proxemic preferences changed to improve the robot's understanding of human social signals. An experiment attenuated a robot's minimum, maximum, and average performance with respect to distance. Participants $(N=180)$ instructed a robot using speech and pointing gestures, provided their proxemic preferences before and after the interaction, and then responded to a questionnaire.

Two major findings are reported with respect to behavioral measures. First, people are predictably poor at estimating distance to the location of robot peak performance; the relationship between participant perceived and actual distance to the location of peak performance is represented well by a power law (described in Section 6.1.2. Second, people adjust their proxemic preferences to be near the perceived location of maximum robot understanding (described in Section 6.1.3.

We also report significant relationships between automated robot performance rates (maximum, minimum, and average) and user subjective measures of autonomous sociable robot competence, anthropomorphism, engagement, likability, and technology adoption. Our results provide quantiative evidence for what has to date been intuitive/anecdotal: objective robot performance-rather than superficial factors, such as robot appearance (Walters, 2008) and height (Hiroi \& Ito, 2011; Rae et al. 2005) — can be a determining factor in the efficacy of autonomous sociable robots. Contrary to related work, a lack of evidence was found to support the notion that human-robot proxemics is a 
significant predictor of commonly investigated factors, such as user-perceived safety, comfort, and likability (Henkel et al., 2012; Mumm \& Mutlu, 2011, Takayama \& Pantofaru, 2009).

This work offers insights into the dynamic nature of human-robot proxemics and has significant implications for the design of sociable robots. Considerations and potential approaches for developers to integrate these results into autonomous robot control systems are provided in Section 7 . aiming to promote rich robot autonomy in human-robot interaction.

Traditionally, HRI focuses on ensuring that the robot is meeting the needs of the user. The impact on the robot itself is often an afterthought. Our results suggest that even novice users are willing to adapt their behavior in an effort to help the robot to better understand and perform its tasks. This is of key importance, since automated recognition systems are unlikely to be perfect, yet that is not a reason to delay the development, deployment, and benefits of social and socially assistive robots. Robots have needs too-and human users will identify, accept, and attempt to meet them.

\section{Acknowledgments}

This work was supported in part by an NSF Graduate Research Fellowship, the NSF National Robotics Initiative (IIS-1208500), and NSF grants CNS-0709296 and CNS-1513275.

We thank Aditya Bhatter, Lizhi Fan, Jonathan Frey, Chloe Grubb, Edward Kaszubski, Erika King, Lixing Liu, Akash Metawala, Kedar Prabhu, and Cherrie Wang for their assistance in developing the experimental systems and setup, recruiting participants, and conducting the experiment.

\section{References}

Argyle, M., \& Dean, J. (1965). Eye-contact, distance, and affliciation. Sociometry, 28, 289-304. doi:10.2307/ 2786027

Bartneck, C., Croft, E., Kulic, D., \& Zoghbi, S. (2009). Measurement instruments for the anthropomorphism, animacy, likeability, perceived intelligence, and perceived safety of robots. International Journal of Social Robotics, 1(1), 71-81. doi:10.1007/s12369-008-0001-3

Breazeal, C. (2003). Social interactions in hri: The robot view. IEEE Transactions on Man, Cybernetics, and Systems, 34(2), 181-186. doi:10.1109/tsmcc.2004.826268

Breazeal, C. (2004). Designing sociable robots. Cambridge, MA: MIT Press. doi:10.1016/s0898-1221(03) 80129-3

Eresha, G., Haring, M., Endrass, B., Andre, E., \& Obaid, M. (2013). Investigating the influence of culture on proxemic behaviors for humanoid robots. In Proceedings of the 22nd IEEE International Symposium on Robot and Human Interactive Communication (pp. 430-435). Gyeongju, Korea. doi:10.1109/roman .2013 .6628517

Feil-Seifer, D., \& Matarić, M. (2005). Defining socially assistive robotics. In Proceedings of the 9th IEEE International Conference on Rehabilitation Robotics (pp. 465-468). Chicago, IL. doi:10.1109/icorr .2005 .1501143

Hall, E. (1959). The silent language. New York, NY: Doubleday Company. doi:10.1002/bs.3830070408

Hall, E. (1963). A system for notation of proxemic behavior. American Anthropologist, 65, 1003-1026. doi: 10.1525/aa.1963.65.5.02a00020

Hall, E. (1966). The hidden dimension. Chicago, IL: Doubleday Company. doi:10.2307/1572461

Henkel, Z., Murphy, R., \& Bethel, C. (2012). Towards a computational method of scaling a robots behavior via proxemics. In Proceedings of the 7th ACM/IEEE International Conference on Human-Robot Interaction (pp. 145-146). Boston, MA. doi:10.1145/2157689.2157727

Hiroi, Y., \& Ito, A. (2011). Influence of the size factor of a mobile robot moving toward a human on subjective acceptable distance. Mobile Robots-Current Trends, 177-190. doi:10.5772/26512

Kahn, P. (2011). Technological nature: Adaptation and the future of human life. Cambridge, MA: MIT Press. doi:10.7721/chilyoutenvi.22.1.0319 
Mead, R., Atrash, A., \& Matarić, M. J. (2012). Representations of proxemic behavior for human-machine interaction. In Technical Report of the NordiCHI 2012 Workshop on Proxemics in Human-Computer Interaction. Copenhagen, Denmark.

Mead, R., Atrash, A., \& Matarić, M. J. (2013). Automated proxemic feature extraction and behavior recognition: Applications in human-robot interaction. International Journal of Social Robotics, 5(3), 367-378. doi:10.1007/s12369-013-0189-8

Mead, R., \& Matarić, M. J. (2015). Robots have needs too: People adapt their proxemic behavior to improve autonomous robot recognition of human social signals. In Proceedings of the 4th AISB International Symposium on New Frontiers in Human-Robot Interaction. Canterbury, UK.

Mead, R., \& Matarić, M. J. (2016). Perceptual models of human-robot proxemics. Experimental Robotics, 109, 261-276. doi:10.1007/978-3-319-23778-7_18

Mumm, J., \& Mutlu, B. (2011). Human-robot proxemics: Physical and psychological distancing in humanrobot interaction. In Proceedings of the 6th ACM/IEEE International Conference on Human-Robot Interaction (pp. 331-338). Lausanne, Switzerland. doi:10.1145/1957656.1957786

Murata, A. (1999). Basic characteristics of human's distance estimation. In Proceedings of the 1999 IEEE International Conference on Systems, Man, and Cybernetics (Vol. 2, pp. 38-43). Tokyo, Japan. doi: 10.1109/icsmc.1999.825204

Nelder, J., \& Wedderburn, R. (1972). Generalized linear models. Journal of the Royal Statistical Society, 135(3), 370-384. doi:10.2307/2344614

Rae, I., Takayama, L., \& Mutlu, B. (2005). The influence of height in robot-mediated communication. In Proceedings of the 8th ACM/IEEE International Conference on Human-Robot Interaction (pp. 1-8). Tokyo, Japan. doi:10.1109/hri.2013.6483495

Satake, S., Kanda, T., Glas, D. F., Imai, M., Ishiguro, H., \& Hagita, N. (2009). How to approach humans? Strategies for social robots to initiate interaction. In Proceedings of the 4th ACM/IEEE International Conference on Human-Robot Interaction (pp. 109-116). La Jolla, CA. doi:10.1145/1514095.1514117

Short, E., Hart, J., Vu, M., \& Scassellati, B. (2010). No fair!! An interaction with a cheating robot. In Proceedings of the 5th ACM/IEEE International Conference on Human-Robot Interaction (pp. 219226). Osaka, Japan. doi:10.1109/hri.2010.5453193

Stevens, S. (2007). On the psychological law. Psychological Review, 64, 153-181. doi:10.1037/h0046162

Takayama, L., \& Pantofaru, C. (2009). Influences on proxemic behaviors in human-robot interaction. In Proceedings of the 2009 IEEE/RSJ International Conference on Intelligent Robots and Systems (pp. 5495-5502). St. Louis, MO. doi:10.1109/iros.2009.5354145

Torta, E., Cuijpers, R. H., \& Juola, J. F. (2013). Design of a parametric model of personal space for robotic social navigation. International Journal of Social Robotics, 5(3), 357-365. doi:10.1007/s12369-013 $-0188-9$

Torta, E., Cuijpers, R. H., Juola, J. F., \& van der Pol, D. (2011). Design of robust robotic proxemic behaviour. In Proceedings of the 3rd International Conference on Social Robotics (pp. 21-30). Amsterdam, Netherlands. doi:10.1007/978-3-642-25504-5_3

Walters, M. (2008). The design space for robot appearance and behaviour for social robot companions. Hertfordshire, UK: University of Hertfordshire.

Walters, M., Dautenhahn, K., Boekhorst, R., Koay, K., Syrdal, D., \& Nehaniv, C. (2009). An empirical framework for human-robot proxemics. In Proceedings of the 1st AISB International Symposium on New Frontiers in Human-Robot Interaction (pp. 144-149). Edinburgh, Scotland.

Walters, M., Syrdal, D., Koay, K., Dautenhahn, K., \& te Boekhorst, R. (2008). Human approach distances to a mechanical-looking robot with different robot voice styles. In Proceedings of the 17th IEEE International Symposium on Robot and Human Interactive Communication (pp. 707-712). Munich, Germany. doi:10.1109/roman.2008.4600750

Ross Mead, Interaction Lab, University of Southern California, Los Angeles, CA, USA. Email: rossmead@usc.edu. Maja J Matarić, Interaction Lab, University of Southern California, Los Angeles, CA, USA.Email: mataric@usc.edu. 